\title{
THE INTEGRATED DECISION MODEL IN EMERGENCY DISPATCH MANAGEMENT AND ITS IMPLICATIONS FOR DESIGN
}

\author{
B.L. William Wong \\ Department of Information Science, University of Otago \\ Dunedin. New Zealand. \\ email: william.wong@stonebow.otago.ac.nz \\ http://divcom.otago.ac.nz/infosci/msrl
}

\begin{abstract}
This paper reports on a review of a number of well-known naturalistic decision making. NDM, models (Zsambok \& Klein, 1997). Key features of these models were identified and were found to represent different views of the same naturalistic decision making process. The key features of these different views were then integrated into a single model called the Integrated Decision Model. To validate the model, a study of emergency dispatch co-ordinators was conducted to determine the 'goodness of fit' of the model in a naturalistic domain. A Critical Decision Method-based study (Klein et al., 1989) was conducted and found enough evidence in the emergency dispatch management process to support the proposal of the integrated Decision Model.
\end{abstract}

\section{KEY WORDS}

Naturalistic decision making, cognitive engineering, emergency dispatch management, integrated decision model.

\section{INTRODUCTION}

One of the primary concerns in Cognitive Engineering is the understanding of how operators make decisions about their tasks in various dynamic human-machine work domains such as nuclear power plants, and manufacturing (Rasmussen et al., 1994; Vicente, 1992). Such domains have generally been classificd as causal domains (Rasmussen, 1986), i.e. domains in which the outcomes are predictable by the laws of nature. Naturalistic decision making (NDM) research has focused on how operators make decisions in dynamic human activity systems such as military and emergency command and control (Drillings \& Serfaty, 1997; Kaempf et al., 1996; Wong, 1999). Such domains have generally been classified as intentional domains (Rasmussen, 1986), i.e. domains where the outcomes are not predictable by the laws of nature, but are influenced by the motivations of individuals in the process. Despite differences in their areas of focus, both fields of research have studied decision making in a number of actual situations and have explored how this might affect the manner in which information about the work domain should be portrayed (Klein, 1993; Vicente et al., 1995; Wong et al., 1998). This has lead to the development of a number of decision models which differ in certain key characteristics depending upon the perspective of the various researchers. These models thus represent different views of decision making in such dynamic work domains.

This paper will summarise the key features of a number of such decision models and attempt to integrate them into a single model that incorporate all the key features of decision making in dynamic decision making domains. This model has been referred to as the Integrated Decision Model, or IDM (Wong, 1999). Based on a grounded theory approach, a study was undertaken to identify the decision goals and stratcgies of emergency ambulance dispatchers at the St John's Ambulance Southern Regional Communications Centre in Dunedin, New Zealand, and at the Ambulance Co-ordination Centre of the Ambulance Service of New South Wales in Sydney (Wong et al., 1996; Wong et al., 1995). The strategies were evaluated in the light of the key features of the NDM models and enough evidence was found to support the proposition that the Integrated Decision Model can usefully bring together and collectively demonstrate the different perspectives in a single decision model. The usefulness of the IDM to interface design is that it reinforces the need for displays to support situation awareness and situation assessment through pattern matching and recognising, rather than by analytical strategies that require option generation and simultaneous evaluation of multiple options to select an optimal solution.

The next section will summarise the key features of a number of decision models and then describe the Integrated Decision Model. The following section will briefly report on the key findings from a study that elicited evidence from dispatchers that provide support for the proposed IDM. Then the final section will discuss some implications that the IDM may have for the design interfaces to dynamic processes.

\section{KEY FEATURES OF NATURALISTIC DECISION MODELS}

NDM environments have been found to exhibit the following characteristics (Orasanu \& Connolly, 1993):

a. Ill-structured problems where the causes are not clear or obvious.

b. Uncertain, dynamic environments that are constantly changing.

c. Shifting, ill-defined, or competing goals due to the changing situation and competing needs.

d. Action/feedback loops where outcomes of an earlier decision have an impact on downstream decisions. 
e. Time stress.

f. High stakes, e.g. possible loss of life.

g. Multiple players that need to co-ordinate actions and decisions.

h. Organisational goals and norms that constrain actions.

Several decision models have been developed to describe the nature of decision making in these NDM environments. A number of these decision models were compared to identify their key features: the Decision Ladder (Rasmussen, 1986) the Recognition-Primed Decision Model (Klein, 1997) the Simplified Decision Model (Orasanu \& Fischer, 1997) the Situation Awareness model (Endsley, 1995) and the Information Processing model of decision making (Wickens, 1992). These key features are summarised below.

\section{Situation assessment an important part of decision making}

A situation assessment is a conceptualisation or mental model of what is happening and is likely to happen in an environment. Decision makers are said to be situationally aware if they have a mental model that is an up-to-date representation of the situation. The models reviewed described how decision makers in dynamic environments place a great deal of effort on understanding the situation and what is likely to occur next, rather than on generating and evaluating options. Incomplete or inaccurate situation assessments will lead to actions that may fail to address key aspects of the situation. However, once the problem is understood, courses of action usually become obvious.

\section{Feature matching and story building key to situation assessment}

Situation assessment is said to develop through either a feature matching or a story building strategy. The feature matching strategy is based on the pattern-recognition of cues in the current situation. When the situation is recognised to be sufficiently similar to previous experiences, appropriate actions that satisfy the condition-action rules are selected and implemented. However, when the patterns of activity in the situation are not sufficiently similar to past experiences, and therefore fail to activate a relevant schema, the story building strategy is invoked to construct a causal explanation of the current circumstances. Story-building techniques are also used by decision makers to cope with incomplete and uncertain data. By drawing on previous experiences, knowledge and expertise (schemata), decision makers make inferences about the data by filling in gaps created by the incomplete data. The resulting understanding of the situation leads to the selection of an appropriate set of actions.

\section{Situations are not presented in an optimal manner}

The cues of a situation are often presented through different sources and over a period of time. How the cues are pieced together will influence how the situation is understood, the conclusions that can be drawn, and hence the choices that are made. Human biases, e.g. anchoring, are to be expected. Designs need to anticipate and avoid the likelihood of such biases.

\section{Option generation: multiple vs. single}

Decision makers in dynamic environments do not have the time to analytically generate multiple options. They do not ask the question, "What are the likely ways to solve the problem?" Instead, they seek to identify the actions that best match the pattern of activities recognised in the situation assessment, one option at a time.

\section{Serial simulation, not concurrent analytical evaluation}

In contrast with analytical decision theory, the NDM models suggests that decision makers serially evaluate courses of actions by pattern-recognition rather than by comparing the strengths and weaknesses of multiple options in parallel. In terms of the very short time horizon (seconds and minutes), decision makers simply do not have the time to generate multiple options and then to analytically evaluate them. If the decision maker has some extra seconds available, he or she may choose to re-evaluate the situation to see if there are late changes in the situation, or to fine-tune the course of action. Courses of action are directly implemented because they satisfy the required condition-action rules. If there is any doubt that the course of action will satisfy the situation, it can be mentally rehearsed to determine if it will work. Modifications are made to the plan and the actions are implemented. If, however time is not available, the closest match is implemented and the situation monitored and the actions adjusted as the situation develops. This kind of behaviour is very apparent in fast-paced environments such as aviation and emergency dispatch management. 


\section{THE INTEGRATED DECISION MODEL}

Based on the above review, the literature suggests that people in real-world, time pressured environments employ decision making strategies that are different from what have been prescribed by traditional rationality-based decision models, e.g. model of managerial decision making (Simon, 1977). They do not generate multiple options which are then concurrently evaluated on the strengths and weaknesses in order to select an action. Instead, NDM researchers have observed that these people first make a situation assessment by recognising critical cues in the situation, and then using a set of rules, select a course of action by matching the pattern of cues to the first most appropriate action, and then if time permits, serially evaluate and modify the course of action before implementing it. These key features have been brought together in the Integrated Decision Model (Figure 1). The IDM highlights two major processes: A situation assessment process, and a choice process. In assessing what is going on in a situation, the decision maker is presented with various information cues about the situation. Not all cues are attended to due to various factors such as their existing schemata, biases, salience of the cues, and time constraints. The decision maker's schemata of operations feature recognition and pattern matching process is applied to the cues that are perceived. The literature suggests that a story building strategy is invoked to help explain the situation, especially when there is insufficient or incomplete data from which to assess the situation. Decision makers in this manner build up a mental model of the situation. They will use this mental model in subsequent mental rehearsals to verify the appropriateness of action strategies. The selection of these actions is discussed next.

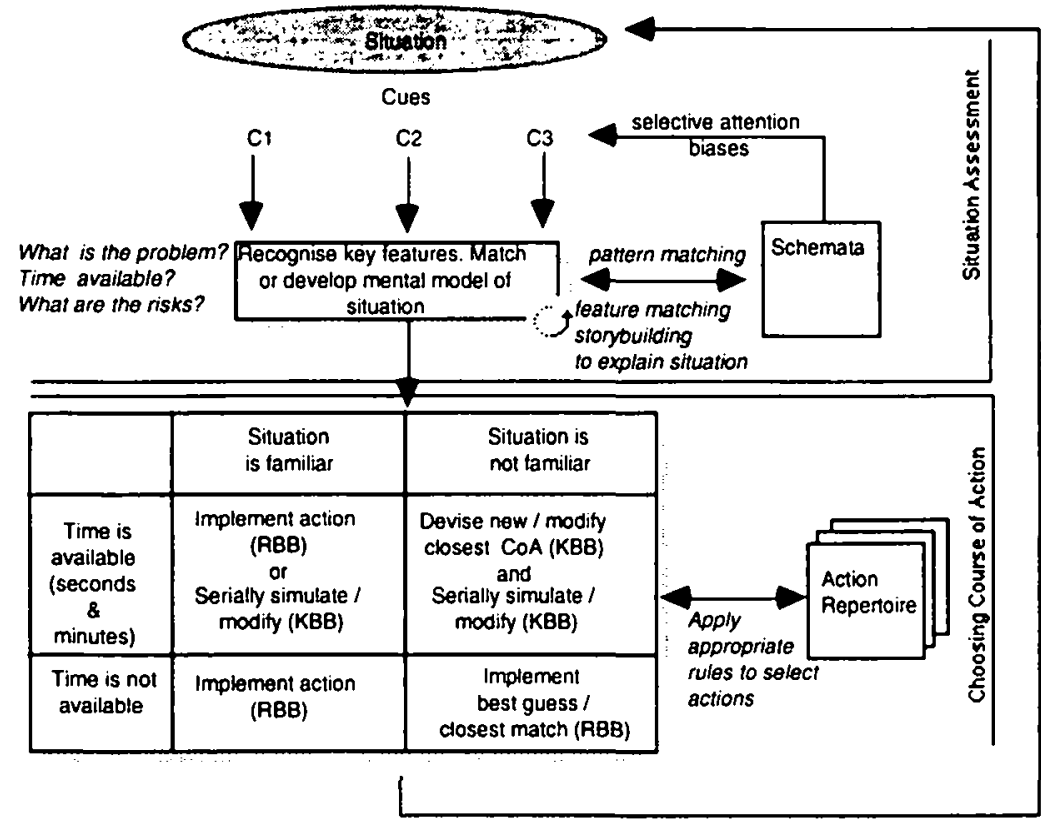

Figure 1: The Integrated Decision Model showing a distinct situation assessment phase and choice phase

Having assessed the situation and updated their mental models of the situation, the decision maker progresses on to the action selection stage of the IDM, 'Choosing Course of Action'. The literature suggests two factors determine how actions are selected: (i) How much time is available, and (ii) How familiar is the situation. These two factors are integrated into a two dimensional action space in the IDM. The four segments of this action space represent:

a. Situations that are familiar and when time (in terms of seconds and minutes) is available. If the situation is one which the decision maker has had experienced with, he or she will apply a rule-based behaviour (RBB) strategy and implement an action that satisfies a given rule set. If however the situation is familiar but the same as previously experienced ones, and hence there is only a partial match between the cues of the situation and the rule set, the decision maker will then embark on a knowledge-based behaviour (KBB) mental simulation strategy to serially test and modify existing actions for which the situational cues and rules do not fully match (see Sanderson \& Harwood, 1988) for a good discussion of skill-, rule-, and knowledge-based behaviours.)

b. Situations that are familiar but time is not available. The decision maker will apply the action most closely reflect actions that have worked in the past with the view of correcting and compensating once the outcomes of those actions are known.

c. Situations that are not familiar and time is available. In such situations, the decision maker will have the time to devise new courses of action and to then modify them. Such an action development strategy is 
typical of knowledge-based behaviour patterns. A serial evaluation of other actions for possible fit in the current situation is also undertaken.

d. Situations that are not familiar and time is also not available. The selected action is usually to implement the best guess based on the closest match between the situational cues and the rule set for similar types of situations.

Evidence was then sought to verify whether such a model could describe decision making behaviour in emergency dispatch management. The next section presents some findings from such a study.

\section{EVIDENCE FOR SUPPORTING THE INTEGRATED DECISION MODEL}

A retrospective protocol analysis technique called the Critical Decision Method (Klein et al., 1989; Wong et al., 1997) was used to elicit decision strategies of emergency ambulance dispatchers at ambulance control centres in Dunedin, New Zealand, and Sydney, Australia. The protocol analysis revealed evidence that supported the IDM. Key findings from this study are summarised below. Transcripts that support the key findings have not, however, been included here due to space constraints, but have been reported in detail elsewhere (Wong, 1999).

\section{Intense and Time Constrained Environment}

One situation that is representative of the intense and time constrained nature of work in the RCC was selected for further investigation. This part of the study compared audio tapes of operations with computer print-outs of events that occurred during that period. The workload in the first nine minutes of the situation was so intense that many of the events that occurred were not documented in the computer until later. During that short span of 9 minutes, a total of 10 emergency calls were received by two duty officers. The calls related to three major road traffic accidents and one attempted suicide. During this initial nine minutes, the dispatchers had to answer the calls, diagnose the problems, type in the details of the call, distinguish between multiple calls about the same incident, plan the response, and then co-ordinate with the six emergency ambulances.

\section{Ambiguous, Ml-defined and Unstructured Problems}

Analysis of the transcripts show that the problems faced by the dispatchers are largely ill-defined and unstructured. Callers are frequently ambiguous in describing the problem and even the location. The cause of ambiguity can be attributed to caller incoherence because "... he was so hyped up." (\#4/226), or it can be due to information arriving through secondary sources at different times. The situation is often further complicated by unclear symptoms reported by the caller. Dispatchers have found it necessary to control the caller's conversation in order to reduce this ambiguity. Failing to clearly diagnose the problem or to determine where it occurs, leads to two major difficulties: Dispatchers cannot devise an appropriate plan, and they cannot dispatch any ambulances. Failing to correctly ascertain the location will lead to major errors in dispatch, delaying the delivery of medical assistance, and wasting a resource. The actual dispatch task is often straightforward, send an ambulance to a particular address.

\section{Recognition-Primed Situation Assessment}

Through their training and experience, dispatchers build up a schemata of problem situations. These schemata provides the basis for recognising the type and severity of situations (Lipshitz \& Ben Shaul, 1997). It is with these schemata that dispatchers compare presented cues of a new situation to diagnose what is wrong. Familiarity with or a recognition of the situation allows a quick translation of the severity assessment into the number of ambulances or type of resources needed. This is usually a straightforward calculation based on the carrying capacity of each type of ambulance. Identifying which ambulances to send is the problem. This is discussed next.

\section{Looking for the Nearest Available Ambulances}

Once it is clear what the requirement for ambulances are and where the ambulances should go, the dispatcher next need to identify and send the nearest available ambulances. Several strategies for identifying the nearest available ambulances have been identified through the CDM interviews. These strategies are (a) ask the caller, (b) areas of responsibilities, (c) diversion of non-critical job ambulances, and (d) focus and compare. While the first three strategies are somewhat self-explanatory, the focus and compare strategy requires some explanation. In deciding which ambulances to send, the dispatcher mentally combines two types of information: location of the incident, and location of the stations to determine the nearest ambulances. Having determined which stations are closest to the incident, the dispatcher focuses and eliminates from consideration all other possible stations that are further away from the accident. He focuses attention only on the ambulance stations that are relevant to the situation. The dispatcher then compares between the candidate stations and dispatches the most appropriate ambulances based on availability and crew qualification. 


\section{Sequentially Develop and Evaluate Courses of Action.}

Dispatchers do not appear to generate multiple options which are then evaluated and from which the most suitable option is selected. Based on the information presented to him, the dispatcher generates one single option, i.e. send the closest ambulance. If necessary, this strategy is modified to work within the circumstances of the situation. If the first course of action was unworkable, based on her situation awareness and where the incident is, the dispatcher mentally rehearses and modifies the plan in her head.

\section{Balancing ambulance coverage.}

Whenever ambulance coverage is depleted in one region due to a high level of activity or due to a major incident, dispatchers still need to provide ambulance cover for those communities. They do this by sharing the coverage between depleted regions and regions that still have ambulances. Coverage between those regions that have ambulances and those that do not is balanced by re-deploying ambulances in locations that will allow them to respond to emergencies in either region, although with a degraded response time. This is described as the 'snowball effect'. They move the closest available ambulance nearer to the depleted zones, and then move the next nearest available ambulances closer again to cover the gaps created, and so on.

\section{Situation A wareness}

Another important aspect of the decision process that is not usually identified through a traditional systems analysis (e.g. data flow diagram) is that of situation awareness. Endsley has described situation awareness as the state of knowing what is going on in one's area of interest (Endsley, 1995). Although not reported throughout the interviews it was observed that dispatchers found it necessary to build and maintain a good mental picture of where each ambulance generally is and what each is doing. The status screen provides a quick reference to confirm their mental picture of what is going on in the region.

\section{THE DECISION PROCESS IN TERMS OF THE IDM}

The various aspects of the decision process identified through the CDM interview may be viewed in the context of the Integrated Decision Model. This is presented in Figure 2. The caller provides the problem cues from which the dispatcher assesses in terms of his schemata of problem situations. He assesses the seriousness of the incident. Understanding what the problem is and where it is, leads the dispatcher to know what ambulances need to be sent and where it is to be sent. This is the assessment of the situation. If the situation is familiar, the dispatcher simply responds by selecting an action based on a given set of rules, e.g. look for the nearest available ambulance and send it If it is not familiar or that the requirement cannot be satisfied easily, alternative courses of action to balance the region or to organise a multi-ambulance response, are generated and evaluated sequentially. The characteristics of dispatch management as described above compares favourably with the proposed Integrated Decision Model.

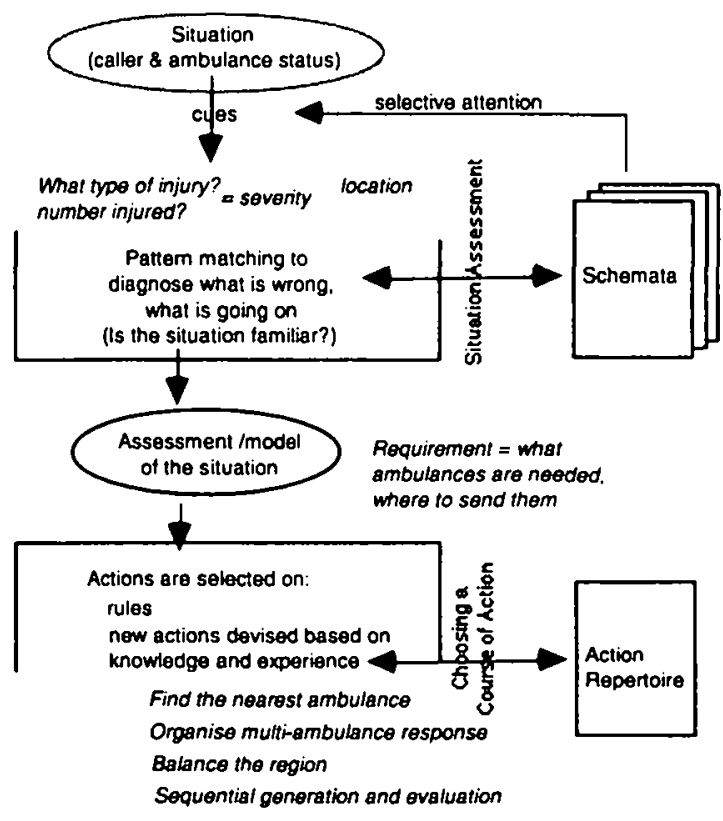

Figure 2 Emergency ambulance dispatch management in terms of the IDM 


\section{IMPLICATIONS FOR INFORMATION PORTRAYAL DESIGN}

\section{Support situation assessment}

Since situation assessment plays such an important role in decision making, displays should portray information about the situation, and where possible show relationships that help explain the situation.

\section{Accessibility of information}

Task-critical information should be easily accessed, at the fingertips or displayed on system. The dispatcher should not have to spend more time than is necessary, or be engaged in protracted procedures to retrieve information required by the decision. For example, in the current system, the dispatcher has to work through five different computer menu changes in order to access the screen that gives directions on how an ambulance might travel to a particular address.

\section{Focus and Compare Strategy}

Dispatchers were observed to practise the focus and compare strategy in a number of situations to locate or confirm the nearest available ambulances. These situations include planning for multi-vehicle responses, balancing the regions, and for maintaining situation awareness. In the focus and compare strategy the dispatcher focuses on a subset of related stations to compare the availability of ambulances for an incident that has occurred in or around the vicinity of the stations. The Proximity-Compatibility Principle (Wickens \& Carswell, 1995) predicts that if information is used together in the making of a decision (task proximity), then especially in time-constrained environments, it will benefit the decision maker to have task relevant information displayed together (display proximity). The information used to determine which ambulances to dispatch to an incident are, according to the PCP, non-integrative in nature and are functionally and temporally similar. This means that the information serves a common goal, is used together or combined at the same time for the same task, but is not integrated into a common variable. This represents a high degree of task proximity. Therefore, in order that the display is compatible with the focus and compare strategy, it is necessary to portray these pieces of information in a manner that exhibits high display proximity, i.e. close together and in a manner compatible with the mental strategy of the task.

\section{CONCLUSION}

This paper has proposed the Integrated Decision Model of naturalistic decision making in an attempt to streamline what appears as parallel description of the same phenomena. A study was done to determine if the proposed IDM can be used to explain behaviour in dynamic decision making environments, in particular, emergency ambulance dispatch management. While there appears to be adequate evidence to suggest that the IDM can be used to explain decision making behaviour in NDM environments, further work is being planned to verify the model and its usefulness for design.

\section{REFERENCES}

Drillings, Michael, \& Serfaty, Daniel. (1997). Naturalistic Decision Making in Command and Control. In G. K. Caroline E. Zsambok (Ed.), Naturalistic Decision Making (71-80). Mahwah, NJ: Lawrence Erlbaum Associates, Publishers.

Endsley, Mica R. (1995). Toward a theory of situation awareness in dynamic systems. Human Factors, 37(1), 3264.

Kàempf, George L., Klein, Gary, Thordsen, Marvin, \& Wolf, Steve. (1996). Decision making in complex naval command and control environments. Human Factor (Special Issue),.

Klein, Gary. (1993). Naturalistic Decision Making: Implications for Design (SOAR 93-1): Crew Systems Ergonomics Information Analysis Centre, Wright-Patterson AFB, USAF, OH.

Klein, Gary. (1997). The Recognition-Primed Decision (RPD) Model: Looking back, looking forward. In G. K. Caroline E. Zsambok (Ed.), Naturalistic Decision Making (285-303). Mahwah, NJ: Lawrence Erlbaum Associates, Publishers.

Klein, Gary A., Calderwood, Roberta, \& Macgregor, Donald. (1989). Critical decision method for eliciting knowledge. IEEE Transactions on Systems, Man and Cybernetics, 19(3), 462-472.

Lipshitz, Raanan, \& Ben Shaul, Orit. (1997). Schemata and mental models in Recognition-Primed Decision Making. In G. K. Caroline E. Zsambok (Ed.), Naturalistic Decision Making (293-303). Mahwah, NJ: Lawrence Erlbaum Associates, Publishers.

Orasanu, Judith, \& Connolly, Terry. (1993). The re-invention of decision making. In G. A. Klein, J. Orasanu, R. Calderwood, \& C. E. Zsambok (Eds.), Decision Making in Action: Models and Methods (3-20). Norwood, NJ: Ablex Publishing Corp. 
Orasanu, Judith, \& Fischer, Ute. (1997). Finding decisions in natural environments: The view from the cockpit. In G. K. Caroline E. Zsambok (Ed.), Naturalistic Decision Making (343-357). Mahwah, NJ: Lawrence Erlbaum Associates, Publishers.

Rasmussen, Jens. (1986). Information Processing and Human-Machine Interaction: An Approach to Cognitive Engineering. (Vol. 12). New York: Elsevier Science Publilshing Co., Inc.

Rasmussen, J., Pejtersen, A.M., \& Goodstein, L.P. (1994). Cognitive systems engineering. New York: Wiley.

Simon, H.A. (1977). The new science of management decision. Englewood Cliffs, NJ: Prentice Hall.

Vicente, Kim J. (1992). Multilevel interfaces for power plant control rooms I: An integrative review. Nuclear Safety, 33(3), 381-387.

Vicente, Kim J., Christoffersen, Klaus, \& Pereklita, Alex. (1995). Supporting operator problem solving through Ecological Interface Design. IEEE Transactions on Systems, Man, and Cybernetics, 25(4), 529-545.

Wickens, Christopher D. (1992). Engineering psychology and human performance. (2nd ed.). New York, NY: Harper Collins Publisher.

Wickens, Christopher D., \& Carswell, C. Melody. (1995). The Proximity Compatibility Principle: Its psychological foundation and relevance to display design. Human Factors, 37(3), 473-479.

Wong, B.L. William. (1999). Information Portrayal in Dynamic Decision Making Environments. Unpublished PhD thesis, Department of Information Science, University of Otago, Dunedin, NZ.

Wong, William B.L., O'Hare, David, \& Sallis, Philip J. (1996). A Goal-Oriented Approach for Designing Decision Support Displays in Dynamic Environments. In J. Grundy \& M. Apperley (Eds.), Proceedings of $\mathrm{OzCHI}$ '96, The Sixth Australian Computer Human Interaction Conference, November 24-27, 1996, Hamilton, New Zealand, 78-85.

Wong, William B.L., O'Hare, David, \& Sallis, Philip J. (1998). The Effect of Display Location on Decision Making in Dispatch Management. In H. Johnson, L. Nigay, \& C. Roast (Eds.), People and Computers XIII, HCI '98 Conference (221-238). Sheffield, UK: Springer, in collaboration with the British Computer Society.

Wong, William B.L. , Sallis, Philip J. , \& O'Hare, David. (1995). Information Portrayal for Decision Support in Dynamic Intentional Process Environments. In H. Hasan \& C. Nicastri (Eds.), OZCHI '95, The Fifth Australian Conference on Computer Human Interaction (43-48). University of Wollongong, Wollongong, Australia: Computer Human Interaction Special Interest Group of the Ergonomics Society of Australia.

Wong, William B.L., Sallis, Philip J., \& O'Hare, David. (1997). Eliciting information portrayal requirements: Experiences with the Critical Decision Method. In H. Thimbleby, B. O'Conaill, \& P. Thomas (Eds.), People and Computers XII, HCI ' 97 Conference of the British Computer Society Special Interest Group on HumanComputer Interaction (397-415). University of West England, Bristol, UK: Springer.

Zsambok, Caroline E., \& Klein, Gary (Eds.). (1997). Naturalistic Decision Making. Mahwah, NJ: Lawrence Erlbaum Associates, Publishers. 